\title{
Endoscopic treatment of gastro esophageal reflux disease: a review of techniques currently in use the novelty of GERD-X
}

\section{Introduction}

Gastric reflux causes symptoms related to irritation of the esophagus and larynx. It is named gastro-oesophageal and gastrolaryngeal reflux. These symptoms are caused by acid regurgitation, or alkaline or mixed, which come from the stomach and the duodenum.

Often, the symptoms did not match documented tissue's damage: this is called N.E.R.D. (Not Esophagitis Reflus Disease). The quality of life is highly invalidated by this disease, but gastroscopy shows an almost normal finding. Only incontinence or beanza cardia determine the major hassles that plague the patient.

\section{Epidemiology and Pathogenesis}

The incidence of reflux disease or Gastro-Esophageal or GastroLaryngeal reflux reaches up to over $50 \%$ of the general population and natural history includes periods of wellness, alternating with periods of exacerbation. The flat mucosa of esophagus is not designed to withstand the irritating contents, which come from the stomach, and this creates problems, with or without tissue damage associated. Erosions and ulcers may occur, with the possibility of a transformation of the esophageal tissue in the sense metaplastic (Barrett's esophagus) and dysplastic, which is a precancerous lesion. For this reason, the endoscopic surveillance should be constant.

The clinical presentation is often manifested with cardiac, neurological symptoms, ENT and pneumological, such as palpitations, chest pain, headache, cough, laryngitis, burning mouth, otitis, bronchopathies, sense of suffocation, even in the absence of the classic heartburn.

\section{Treatment}

The Proton pump inhibitors (drugs that reduce stomach acid) are very often the solution of the problem, because the pill in the morning drastically reduces the symptoms. Recent research, however, attribute to these drugs major side effects, of which it was given less importance in the past. Their continued use would promote intestinal and bronchopulmonary infection, for reducing acid barrier. These drugs can cause hypochromic anemia, reduced absorption of folate and iron, can reduce serum calcium and magnesiemia, with subsequent catabolic processes of the bone and risk of pathological fractures.

\section{Non-drug therapies}

Results from the scientific literature and from my own experience that these drugs have a decreasing effectiveness, not because they give habituation over time, but because the clinical presentation of the disease is often complex. There is a motor disorder, a visceral yperalgesia and a sphincter apparatus malfunction. The "alternative" treatments, which sometimes I advise to my patients, are becoming increasingly important, and allow to reduce, significantly, the intake of drugs. The global postural physical therapy acts on the diaphragm and on the apparatus sphincter cardia and
Volume 6 Issue I - 2017

\author{
Antonio lannetti \\ Department of Medicine, University of Rome, Italy
}

Correspondence: Antonio lannetti, Department of Medicine, University of Rome, Italy, Email antonio.iannetti@uniroma l.it

Received: November 12, 2016 | Published: January 29, 2017

obtains, in some cases, studied and selected, a positive outcome. The pharmacological treatments favors, at times, psychoactive drugs, which act on the enteric nervous system and motility. Patients complain of chronic dependence from taking daily medication and express their intent to seek a permanent solution and not pharmacological.

The surgery has recovered to some extent in the therapeutic indications, with the possibility of being performed laparoscopically, with very reduced recovery time. The introduction of some technical innovations, such as a magnetic necklace Lynx, contributes to the indication for surgery. The ring is placed around the esophagus with a laparoscopic procedure, and does not alter the apparatus of esophageal-gastric sphincter structure. It is still being conducted to test the effectiveness of the procedure.

But, above all, new impetus have the surgery Endoscopic techniques, which aim to strengthen the esophageal-gastric sphincter apparatus, with endoluminal fundoplication, that is performed during a gastroscopy.

Both the choice, Endoscopic and Surgical choice, require a prior anatomical and pathophysiological study of the stomach and esophagus. After clinical assessment and gastroscopy, for anatomical study, you should run the ph- Impedenzio metry of 24 hours and esophageal manometry. The latter two tests are needed, because they document the real cardial incontinence and demonstrate the correlation between disorders and episodes of reflux.

\section{Endoscopic therapy}

In recent years, many endoscopic methods have been developed, for a conservative treatment, aimed at patients not respoder to medical therapy and in patients for whom it was preferable to a nonpharmacological treatment. These procedures are simple, economical and repeatable. They include technical of intraluminal suture, the radio-ablation (STRETTA procedure) and injection of inert substances not absorbable.

\section{STRETTA procedure}

STRETTA procedure consist in putting some needle electrodes in the distal esophagus, applying on these of radio frequency energy, which burns tissue, creating a reactive fibrosis, making it insensitive to algogenic stimuli. This method is used mainly for treating "Barrett's 
esophagus", because it burns the tissue and eliminates the diseased mucosa.

\section{Endoscopic injection of mass agents}

The endoscopic injection of "bulking agents" was used in the past and is still performed in some American countries. It has discrete effects in controlling symptoms, in the short and medium term, improving the manometric findings. Several substances were used: bovine collagen, polietilenmetacrilato (Plexiglas), the ethylene-vinyl alcohol polymer (Enterix), but some have been withdrawn from the market due to adverse events. The injection of foreign material can cause inflammation and ischemia of the esophageal mucosa. They have also been described remote reactions, such as embolizations, in the case dell'Enteryx. Therefore, this method was abandoned.

\section{Endoscopic techniques}

The typical endoscopic procedures currently used, are those defined "endoluminal Gastro Plication (ELGP)", which include the Esophyx, the MUSE method, the EndoCinch and GERD-X. They enable the carrying out of the fundoplication being Gastroscopy, identical to the surgical. These endoscopic techniques are possible in selected cases, when the gastric hiatal hernia is not greater than 20 $\mathrm{mm}$ and it has been established the correlation between symptoms and reflux (Figure $1 \& 2$ ).
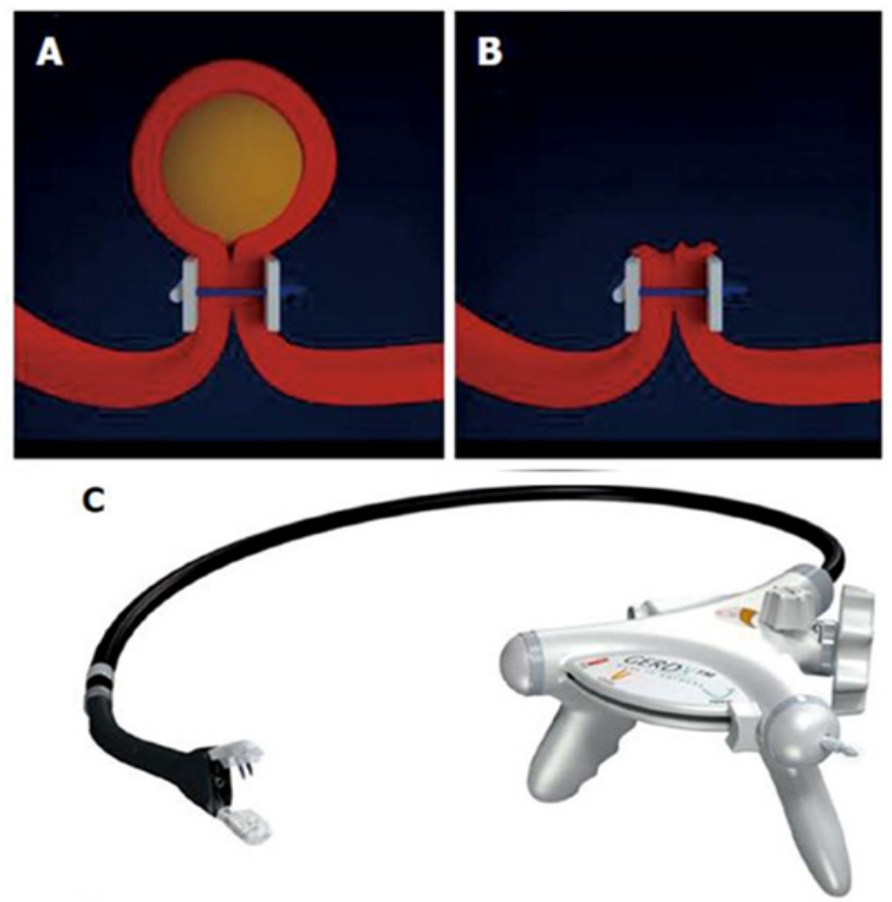

D

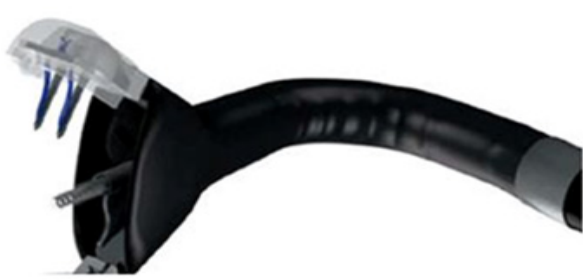

Figure I Detailed RD-X image of the GE: full-thickness plication with platelets (image A).

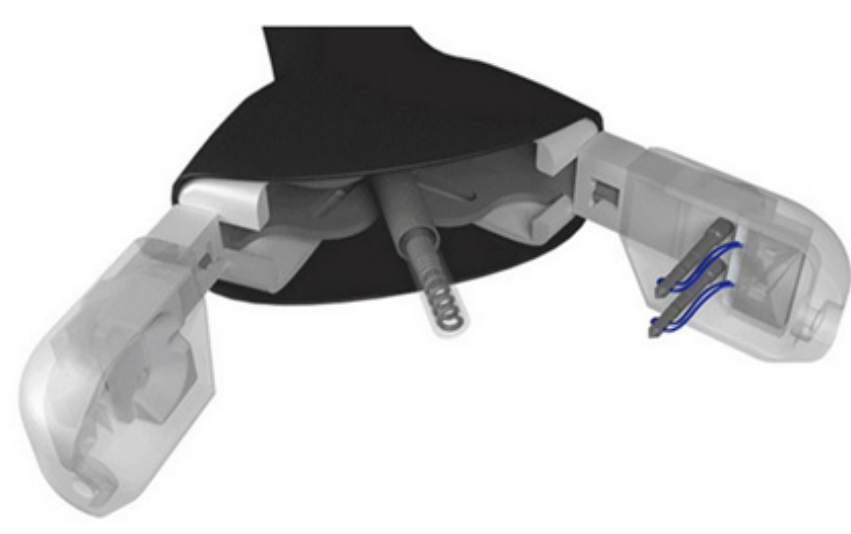

Figure 2 Plicator with platelets at the ends of suture threads.

Esophyx: The Esophyx device creates a fundoplication, suturing the mucous tissue in several places. The operation is performed under vision of a flexible endoscope and oper with double tool.

MUSE: Another endoscopic technique Transoral Incisionless Fundoplication (TIF) is that defined MUSE, Medigus Ultrasonic Surgical Endostapler, which is done with an instrument, equipped with a mini-ultrasound, as well as a stapler. It performs a partial anterior fundoplication. It is equipped with a camera, of the ultrasound probe and a rangefinder. The instrument includes a handle with the controls, a flexible and a rigid section axis, containing a cartridge with five surgical clips.

EndoCinch: EndoCinch is the tool that gives its name to the method and allows to capture, in a niche, located near the tip of the endoscope, the mucosa of the upper stomach, which you want to attach to the mucosa of the sphincter cardia, to form the fundoplication. This mucosa is sutured to form folds and reduce cardia space.

The EndoCinch system provides two instruments and a over-tube. The room, where it is sucked the mucosa of the esophagogastric junction, enables suturing the tissue in a situation of stability, but, despite this, the long-term sealing of the method is not effective. ${ }^{1,2}$

\section{Results}

The initial results of these three methods have been encouraging, but long-term studies have verified the recurrence of symptoms, for the failure of the sutures. Operand endoscopically, are unable to mobilize the gastric fundus, as in surgery, to apply more resistant to traction sutures. In the long run, the traction force exerted on the sutures causes the esophagogastric junction again become incontinent. Even surgical procedures are beset by varying percentages of relapses, sometimes after a short period. But, of course, the present endoscopic techniques, with greater frequency, this problem, in the face of greater ease of execution, of lower costs and ease to again perform the intervention.

\section{GERD-X}

The newer endoscopic technique, GERD-X, which takes its name from stappler endoscopic used, seems to promise a greater durability. This "device" has the advantage of having, at the end of the suture applied, two small plates polyurethane, which make the most solid and stable suture. The success of the intervention is comparable, according to recent scientific studies, carried out in Germany and Austria, to the traditional surgery Nissen fundoplication or Toupet. The follow-up in 
progress are showing that the problem of the reduction of the life, compared to surgery, is passed from the addition of these platelets.

For a chronic condition, as is that of the gastro-oesophageal reflux, the ideal is a simple, cheap surgery, efficient and repeatable. Endoscopic fundoplication (one that is made by gastroscopy) has obvious advantages compared to surgery, that is about the length of stay in Hospital, the execution costs and the ability to repeat the procedure. Basically, you can reduce the hiatus hernia and incontinence of the cardia with a gastroscopy.

The surgery presents a higher percentage of risk, compared to the gastroscopy, the most frequent of which is the esophageal stenosis post-operative. Not to mention the possibility of bleeding and perforations. You need a hospital stay of 4-5 days and it is not always possible to repeat the operation, in case of failure or relapse.

Endoscopy with GERD-X method provides for an intervention in Day Hospital, with a stapler single-use, which is introduced during the gastroscopy, whose advantage is to have a better hold over time, thanks to the two small plates polyurethane, formed from the material used for the hernia networks, which make the suture applied more solid and stable.

This device is the evolution of the NDO Plicator, produced by a company of electromedical Massachusetts, acquired in 2008 by Johnson \& Johnson, which ceased production of Plicator. The Plicator of the NDO Company also had the heads in polytetrafluoroethylene (PTFE), which allow a greater tightness of the suture. The case studies of work of the NDO Plicator has shown good efficacy of these endoluminal suturing in some previous studies in 2008.

The Plicator NDO was a mechanical device and reusable, while the
GERDX has the advantage of a hydraulic mechanism, which does not include blocks, and is disposable, free from bacterial contamination problems (Figure 2).

The risks of this endoscopic procedure are practically zero and, in any case, absolutely lower, compared to those surgical. I refer to the corresponding sections of my professional sites www.iannetti.it and www.gastroenterologoiannetti.com for those who want to know more, both about the clinical presentation of reflux disease, both regarding diagnostic methods, medical therapy with proton pump inhibitors and endoscopic and laparoscopic surgical therapies.

\section{Acknowledgements}

None.

\section{Conflicts of interest}

Authors declare that there is no conflict of interest.

\section{Funding}

None.

\section{References}

1. von Renteln D, Schiefke I, Fuchs KH, et al. Endoscopic full-thickness plication for the treatment of gastroesophageal reflux disease using multiple Plicator implants: 12-month multicenter study results. Surg Endosc. 2009;23(8):1866-1875.

2. UHC. Minimally invasive procedures for gastroesophageal reflux disease (GERD). United Healthcare. Community plan. Policy Number: CS079 D. 2015. 